\title{
Visual field differences in recognizing letters
}

\author{
NORMAN WORRALL \\ Department of Child Development \& Educational Psychology, Institute of Education \\ University of London, 24-27 Woburn Square, London WCIH OAA, England \\ and \\ PETER COLES \\ Department of Experimental Psychology, University of Oxford, Oxford, England
}

\begin{abstract}
Differences due to visual field location were examined for single letters placed at one of 12 clockface positions $3^{\circ}$ and $6^{\circ}$ from fixation. Results compared closely with established findings using simple light stimuli, showing best recognition on the horizontal meridian, poorest on the vertical and intermediate on oblique meridians. Subjects' confidence ratings followed the same pattern. There was evidence for a specific right superiority along the horizontal meridian, as found in previous studies, but no evidence for a general right hemifield superiority. The difficulty of arguing from these findings to available dominance or scanning explanations is pointed out. It is proposed that effects due to visual field variability may have a pervasive yet largely unrecognized influence in visual perception research.
\end{abstract}

The precise location of a stimulus object in the visual field is of considerable importance if we are to know how to interpret data about that object. However, there has been little concern in mainstream psychological research to explore how variations within the visual field might complicate interpretations of results or account for failures to replicate. The standard texts on visual perception stop short at a diagram of the visual pathways-perhaps with the horizontal meridian mapped on to the retinae and an $\emptyset$ sterberg-type plot of relative rod-cone density again along the horizontal meridian. The student may be forgiven for developing, literally, a rather narrow concept of the visual field. Yet there is a wide range of data on visual field differences both between and within meridians. In an early study of the monocular field, for example, Kastner and Wirth (cited in Rains, 1963) showed that bright flashes originating in the left hemifield yielded faster reaction times than those located in the right hemifield: a similar relationship held for upper hemifield flashes with respect to lower hemifield. Rains (1963) and Payne (1966) have developed this work more recently. Size discrimination and the recognition of shapes, letters and complex patterns are all affected by field location (e.g., Pollock \& Chapanis, 1952; Whitmer, 1933), while a number of subject variables such as age and sex have been shown to relate to size of the effective visual field (Burg, 1968; Tronick, 1972).

It is, however, rare for psychological researchers to study visual field differences in the full sense of the term. Target letters in "laterality" studies are almost invariably presented along the horizontal meridian, so that these experiments do not fairly sample a range of locations in the hemifield proper. Studies which have comprehensively sampled the two hemifields, in particular those by Harcum and his colleagues (e.g., Harcum, Note 1) have preferred multiletter or geometric displays which, although yielding interesting findings, introduce other factors such as scanning and order-of-report characteristics. Harcum's work has shown that displays are generally detected/recognized better on the horizontal than on the vertical meridian, with the oblique meridians showing intermediate levels of accuracy. This is in general confirmation of what is shown from clinical perimetry about the organization of the visual field. Thus there is certainly no reason to expect that what may be true for the horizontal meridian regarding letter recognition should also be true for other meridians or more generally for the different hemifields. The purpose of this study was to use a stimulus of accepted psychological interest, letters of the alphabet, to study a phenomenon which we believe should be of rather greater psychological interest, difference in acuity depending on where the stimulus is located in the visual field.

\section{METHOD}

Experiment 1 used eight first-year psychology students, all right-handed and with normal uncorrected vision. A two-field tachistoscope (Electronic Developments) was used to present the target letters, which were printed on white card from Letraset 403, a font without serifs and with all lines of equal thickness. Laterally symmetrical letters were selected: A, O, T, V, W, X. As viewed in the tachistoscope, they were located at one of 12 radially symmetrical positions corresponding to the positions on a clockface. Visual angle from fixation point (in the adaptation field) was $6^{\circ}$, and each letter was approximately $0.5^{\circ}$ in size. Field illumination measured at the viewing aperture was 
Table 1

Mean Recognition Scores (Percentage Correct) for Each Field Position

\begin{tabular}{|c|c|c|c|c|c|c|c|c|c|c|c|c|}
\hline \multirow[b]{2}{*}{$\begin{array}{l}\text { Clock face position } \\
\text { (30-deg steps) }\end{array}$} & \multicolumn{5}{|c|}{$\begin{array}{c}\text { Right Hemifield } \\
\text { (Percentage Correct) }\end{array}$} & \multicolumn{6}{|c|}{$\begin{array}{c}\text { Left Hemifield } \\
\text { (Percentage Correct) }\end{array}$} & \multirow[b]{2}{*}{$\begin{array}{c}12 \\
(\mathrm{VM})\end{array}$} \\
\hline & 1 & 2 & $\begin{array}{c}3 \\
\text { (HM) }\end{array}$ & 4 & 5 & $\begin{array}{c}6 \\
\text { (VM) }\end{array}$ & 7 & 8 & $\begin{array}{c}9 \\
\text { (HM) }\end{array}$ & 10 & 11 & \\
\hline $\begin{array}{l}\text { Experiment } 1 \text { ( } 6 \mathrm{deg} \text { ) } \\
\text { (confidence ratings) } \\
n=8\end{array}$ & $\begin{array}{c}65 \\
(2.3)\end{array}$ & $\begin{array}{l}69 \\
(2.6)\end{array}$ & $\begin{array}{l}84 \\
(3.0)\end{array}$ & $\begin{array}{c}60 \\
(2.4)\end{array}$ & $\begin{array}{l}67 \\
(2.3)\end{array}$ & $\begin{array}{l}60 \\
(2.3)\end{array}$ & $\begin{array}{l}66 \\
(2.5)\end{array}$ & $\begin{array}{c}68 \\
(2.5)\end{array}$ & $\begin{array}{l}78 \\
(2.8)\end{array}$ & $\begin{array}{l}85 \\
(2.6)\end{array}$ & $\begin{array}{c}62 \\
(2.4)\end{array}$ & $\begin{array}{l}49 \\
(2.0)\end{array}$ \\
\hline $\begin{array}{c}\text { Experiment } 2(3 \mathrm{deg}) \\
n=18\end{array}$ & 53 & 59 & 64 & 56 & 45 & 46 & 46 & 53 & 57 & 64 & $62^{\circ}$ & 37 \\
\hline
\end{tabular}

Note-Positions 3 and 9 delineate the horizontal meridian, and 6 and 12 the vertical meridian. In Experiment 1 , percentages are based on 6 (letters) by 2 (runs) by 8 (subjects) =96 trials per position; in Experiment 2, percentages are based on 6 (letters) by 2 (runs) by 18 (subjects) = 216 trials per position. Confidence ratings in Experiment 1 derive from a 4-point scale: Very Sure (1), Fairly Sure (2), Not Sure (3), Guess (4). Note that the smaller percentages overall in Experiment 2 reflect the more difficult criterion (50\% as opposed to $60 \%$ correct on practice trials), and not differences due to distance from fixation.

$0.5 \mathrm{~lx}$. Since the fields in the tachistoscope as supplied were rectangular, circular black masks were fitted over the fields so that each letter was equated for distance from the field border as well as for distance from the fixation point at the center of the adaptation field.

Subjects were told to take their time, on each trial to fixate carefully the center dot in the adaptation field, and then, when ready, to press a button which simultaneously triggered the display field and extinguished the adaptation field. They were told that the display would be a single-letter target in one of 12 "clockface" positions, and that on each trial they were to write down (a) their best estimate of what the letter was; (b) their confidence in the estimate (using a 4-point scale: Very Sure; Fairly Sure; Not Sure; Guess). Exposure time was about $10 \mathrm{msec}$, the exact value being set individually for each subject using a criterion of about $60 \%$ accuracy on a series of initial practice trials. During practice, there was discussion with the subject to ensure as far as possible that he was obtaining a proper fixation before triggering the display. Each subject had 144 trials: 12 (field positions) $\times 6$ (letters) $\times 2$ (runs). A break followed the first run of 72 trials. Experiment 2 used exactly the same apparatus and conditions, save that the stimulus letters were now placed on a $3^{\circ}$ "clockface", 18 subjects were run, and a $50 \%$ instead of a $60 \%$ criterion was adopted in baseline trials: this lower criterion allowed slightly shorter exposure times in an attempt to emphasize field differences already appearing in Experiment 1.

\section{RESULTS AND DISCUSSION}

Inspection of the raw data showed that to a greater or lesser extent, all individual subjects displayed the same trend. It can be seen from Table 1 that recognition was best on the horizontal meridian (clockface position 3 and 9) and worse as the horizontal meridian was, as it were, rotated progressively through $90^{\circ}$. This was true for the results of both experiments. A specific test of trend as the meridian "rotated" gave, for Experiment 1, $z=4.40, p<.01$, and for Experiment 2, $z=5.24, p<.01$ (Ferguson, 1966). It is also evident from the Experiment 1 results that the pattern of confidence ratings followed actual performance quite closely, and confidence trend differences were also significant on the Ferguson test $(z=4.41, p<.01)$. There were large differences in the recognizability of the six letters used: overall, $A$ and $W$ were easiest (both around $85 \%$ accuracy), while $X$ was the most difficult (about $\mathbf{4 0 \%}$ accuracy). However, each letter individually showed the general field position effects evident in Table 1; thus, a distinction can be made between those differences due to field location and those due to central processing factors for more "difficult" alphabetic material, independent of field location. There was no evidence of interaction effects between letter and field location for recognition scores or for confidence measures.

The present data are in good agreement with the evidence from clinical perimetry, and that of Harcum and others, about the relative importance of the horizontal, vertical, and oblique meridians. The data are also in agreement with recent work of Haines and Gilliland (1973), who used a small photopic stimulus to provide a comprehensive map of equal reaction time contours in the visual field. In the introduction to this paper, we briefly indicated the range of field variables relating to stimulus, subject, and task that could affect visual perception experiments. Further examples could be given, such as the finding of Brown (1953) that, "for a given subject and a given part of the visual field, apparent size sometimes varies gradually but markedly with respect to time"-yet another difficulty in the way of obtaining good replication of data, even by the same experimenter using the same subjects on each occasion. But quite apart from the role of field differences as a persistent confounding influence, a case can be made for bringing data on visual field variability more directly into proposed explanations for standard perceptual phenomena. We will try to show briefly how field data could be relevant to two established and contrasting areas of perceptual research, the study of visual short-term storage and the study of visual illusions, then finally we will give a more extended treatment of the relevance of the present data to a third area of research, laterality differences in perception.

Regarding short-term visual storage, one would like to know, for example how storage duration 
estimates are affected by field location. Sperling (1969) makes oblique reference to this when discussing the experiment which used a masking paradigm to determine that the scanning rate for letters was some 10-15 msec per letter: "A serious problem in this kind of experiment is that it is very difficult to ensure that all letters fall upon retinal locations which recover at the same rate." In metacontrast research, the same general difficulty has been recog. nized by Lefton (1973), who concludes that "the problem of retinal location is not fully understood." Eriksen, Collins, and Greenspon (1967) were one group of workers who recognized and attempted to overcome the confounding of degree of metacontrast with field location: they used a circular array with equidistant matching about the central fixation point, but as we have pointed out on the basis of the present data, this procedure does not guarantee stimulus equivalence. In a quite different research area, the study of visual illusions, field effects have been found with the Mueller-Lyer illusion (Clem \& Pollack, 1975; Worrall \& Firth, 1974), and it would be surprising in view of the findings of Pollock and Chapanis (1952) and of Brown (1953) regarding anisotropy of the visual field for line stimuli if this source did not contribute to the misalignment effects of the Poggendorff illusion. This would be particularly likely for the residual misalignment which has been demonstrated in the absence of the vertical parallels (Goldstein \& Weintraub, 1972). In such experiments, where there is typically free eye movement combined with unlimited exposure times, there should be a reduction in the "constant" or "stationary" field effects as found in tachistoscopic procedures. However, the shifting instability superimposed by free inspection could be expected to interact with any inherent instability of the stimulus figure itself, due to nonfield sources such as "lateral inhibition" between particular lines in the figure. On this point, it does seem generally important in visual perception research to distinguish between effects due to line orientation, theoretically linked with orientation detector cells in the visual cortex, and effects due to meridian of orientation, something quite independent. Yet the design of many studies prevents an assessment of how much of the experimental effect is due to a line's being at, say, $45^{\circ}$, and how much is due to its lying along the $45^{\circ}$ field meridian: Appelle (1972), for example, seems to review both kinds of study as being of a single class. Field location effects can, of course, be disengaged from stimulus orientation effects: Thompson and Schiffman (1974), for example, have shown that magnitude of the horizontal-vertical illusion increases as the stimulus figure is moved into the periphery of the visual field.
The third area on which the present findings have some bearing is that of laterality differences in recognizing letters. Although the evidence is not entirely convincing, right hemifield superiority has been claimed when single letters are shown at one of two locations either right or left of fixation (Bryden, 1965; Hayashi \& Bryden, 1967; Heron, 1957). Proposed explanations have included lefthemisphere dominance for verbal materials, a mechanism which scans the persisting trace after the exposure has terminated, and eye acuity dominance. In the present experiments, using single letters and reasonably sampling the two hemifields, there was no evidence of a general right-hemifield superiority. To be accurate, there was, in fact, a slight left superiority at both $3^{\circ} \quad[X($ left $=56.4 \%$; $X($ right $)=55.4 \%]$ and $6^{\circ} . \quad[X($ left $)=71.2 \%$; $X($ right $)=68.4 \%]$. At the same time, in agreement with previous claims, there was evidence for a specific right superiority along the horizontal meridian in both experiments (see Table 1). The conjunction of these two findings carries some significance. In the absence of any general superiority for recognition in the right hemifield, a within-meridian superiority on the right can hardly be explained in terms of "left hemisphere dominance for verbal materials," unless it were wished to make a special case for the projection of the horizontal meridian on to the cerebral hemispheres. Similar special arguments would then be required for the other within-meridian differences in Table 1. Nor are explanations depending on eye dominance (Hayashi \& Bryden, 1967) or hemiretinal dominance (Neill, Sampson \& Gribben, 1971) appropriate unless it is wished again to make a nonparsimonious argument for acuity on the horizontal meridian as opposed to acuity in the hemifield generally. A postexposural scanning explanation of the kind originally proposed by Heron (1957) should be less troubled by inconsistency between field and meridian characteristics, since its derivation-reading along a line of print as an overleaned skill-is more obviously meridian based. However, this kind of explanation is inherently unsuited to explaining the present results, since it is aimed at strings of letters: it is difficult to see in what sense a single letter could be scanned from left to right (see Bryden, 1966; White, 1969). Several explanations of the artifact type have also been proposed to account for field asymmetry. The first kind of artifact concerns rehearsal and order of report (e.g., Smith \& Ramunas, 1971), but these again can have no bearing on the present single-letter displays since there is neither "rehearsal" nor "order." A second kind of artifact, attention bias and/or eyefixation bias, has been proposed by several investigators (e.g., Ayres 1966; Terrace, 1959). However, studies where subjects have actually been told 
beforehand in which hemifield the stimulus would fall (Heron, 1957) or where "forced" and "unforced" instructions to fixate have been compared (Harcum, Hartman \& Smith, 1963) have not suggested any systematic differences due to such sources. Bias explanations seem, in any case, better suited to experiments where only 2 locations are used (rather than 12 locations, as here). To suit the present findings, the bias would again have to be peculiar to the horizontal meridian, and other meridian data would have to be accepted as evidencing different directions of "bias."

It thus seems that there is no "neat" way of accounting for the present disparity between hemifield and hemimeridian data in terms of any single proposed explanation for laterality differences in perception: while several of these explanatory mechanisms may well be relevant, none is sufficient. It is perhaps time someone tried to develop a composite model in which the contribution of several factors could be weighted, although the root difficulty probably arises from oversimplified notions about how hemifields, hemiretinae, and hemispheres somehow link together. The feature of the present data which most fundamentally undermines work to date on letter recognition is the fact that following "good procedure" by equidistant matching about a fixation point does not guarantee stimulus equivalence in the two hemifields; so much is clear from Table 1 . This surely suggests the desirability of bringing visual field data much earlier into theoretical explanations, and the inadvisability of regarding such data as having little more than nuisance value.

\section{REFERENCE NOTE}

1. Harcum. E. R. Visual recognition along various meridians of the visual field: II. Nine-element typewritten targets. Report 2144293-T. Ann Arbor: University of Michigan, 1958.

\section{REFERENCES}

Appelle, S. Perception and discrimination as a function of stimulus orientation: The "oblique effect" in man and animals. Psychological Bulletin, 1972, 78, 266-278.

AYREs, J. J. B. Some artifactual causes of perceptual primacy. Journal of Experimental Psychology, 1966, 71, 896-901.

Brown, K. I. Factors affecting differences in apparent size between opposite halves of a visual meridian. Journal of the Optical Society of America, 1953, 43, 464-472.

BRYDEN. M. P. Tachistoscopic recognition, handedness and cerebral dominance. Neuropsychologia, 1965, 3, 1-8.

Bryden, M. P. Left-right differences in tachistoscopic recognition: Directional scanning or cerebral dominance? Perceptual and Motor Skills, 1966, 23, 1127-1134.
Burg. A. Latera! visual field as related to age and sex. Journal of Applied Psychology. 1968. 52. 10-15.

Clem, R. K., \& Pollack, R. H. Illusion magnitude as a function of visual field exposure. Perception \& Psychophysics, 1975, 17. 450-454.

Eriksen, C. W., Collins, J. F., \& Greenspon, T. S. An analysis of certain factors responsible for non-monotonic backward masking functions. Journal of Experimental Psychology, 1967, 75, 500-507.

Ferguson, G. A. Statistical analysis in psychology and education. London: McGraw-Hill, 1966.

Goldstein, M. B., \& Weintraub, D. J. The parallel-less Poggendorff. Perception \& Psychophysics, 1972, 11, 353-355.

Haines, R. F., \& Gilliland, K. Response time in the full visual field. Joumal of Experimental Psychology, 1973, 53. 289-295.

Harcum, E. R., Hartman, R. R., \& Smith, N. F. Pre- vs. postknowledge of required reproduction sequence for tachistoscopic patterns. Canadian Journal of Psychology, 1963, 17, 264-273.

Hayashi, T., \& BRYden, M. P. Ocular dominance and perceptual asymmetry. Perceptual and Motor Skills, 1967, 25, 605-612.

Heron. W. Perception as a focus of retinal locus and attention. American Journal of Psychology, 1957, 70, 34-48.

Lefton. L. A. Metacontrast: A review. Perception \& Psychophysics. 1973, 13, 161-171.

Neill, D. O., Sampson, H., \& Gribben, J. A. Hemiretinal effects in tachistoscopic letter recognition. Journal of Experimental Psychology, 1971, 91, 129-135.

PAYNe, W. H. Reaction time as a function of retinal location. Vision Research, 1966, 6, 729.732.

Pollack, W. T.. \& Chapanis, A. The apparent length of a line as a function of its orientation. Quarterly Journal of Psychology, 1952, 4, 170-178.

Rains, J. D. Signal luminance and the position effect in human reaction time. Vision Research, 1963, 3, 239-251.

Sмiтh, M. C., \& Ramunas, S. Elimination of visual effect by use of a single report technique: Evidence for order-of-report artifact. Journal of Experimental Psychology, 1971, 87, 23-28.

SPERling, G. A model for visual memory tasks. In R. N. Haber (Ed.). Information processing approaches to visual perception. New York: Holt. Rinehart, Winston, 1969.

Terrace, $H$. The effect of retinal locus and attention on the perception of words. Joumal of Experimental Psychology, 1959. 58. 382-385.

Thompson, J., \& Schiffina, H. R. The effect on magnitude of the horizontal-vertical illusion of horizontal retinal eccentricity. Vision Research. 1974, 14, 1463-1465.

Tronick, E. Stimulus control and growth of the infant's effective visual field. Perception \& Psychophysics, 1972, 11, 373-375.

Whrte, M. J. Laterality differences in perception. Psychological Bulletin, 1969, 72, 387-405.

WhITMER, C. A. Peripheral form discrimination under dark adaptation. Journal of General Psychology, 1933, 7, 405-418.

Worrall, N.. \& Firth. D. E. The components of the standard and reverse Müller-Lyer illusions. Quarterly Joumal of Experimental Psychology, 1974, 26, 342-354.

(Received for publication December 1, 1975; revision received April 21, 1976.) 